\title{
Can Luminol Be a Fluorophore?
}

\author{
M. Fátima Barroso ${ }^{1} \cdot$ Rui J. A. Silva ${ }^{1} \cdot$ Sérgio F. Moreira ${ }^{1} \cdot$ Sofia S. Rodrigues $^{1} \cdot$ Helena M. R. Gonçalves $^{2}$. \\ Abel J. Duarte ${ }^{1}$
}

\begin{abstract}
In this work, we report a new chemiluminescence system using bis-(2,4,6-trichlorophenyl) oxalate (TCPO) with hydrogen peroxide and luminol as fluorophore. The intense chemiluminescence reaction here described was fully investigated and it was determined that this fluorescent system has two strong light emissions at 440 and $490 \mathrm{~nm}$, respectively. This new, user friendly, intense and striking light emission chemiluminescence system can be used as a very usefull tool for the design and construction of fluorescencent chemical sensors.
\end{abstract}

Keywords Chemiluminescence $\cdot$ TCPO $\cdot$ Luminol

\section{Introduction}

Over the years the Chemiluminescence (CL) reaction has been proven quite useful, indeed the application of this chemical process provides a very sensitive, cost-effective detection alternative to many radioisotopic and fluorescence techniques [1].

Chemiluminescence is a phenomenon in which chemicallygenerated molecules emit light in excited states., The CL phenomenon can be described in a simple manner, as a process that occours in two steps: i) The formation of a high excited energetic intermediate and ii) the loss of the intermediate energy by two distinct mechanisms - direct or indirect CL. Direct CL occours when the intermediate relaxes to the ground state by the emission of photons, a well known example of this mechanism, is the oxidation of luminol by the hydrogen peroxide in the presence of a catalyst (Scheme 1a). The aminophthalate ion (Scheme 1a step 2), upon excitation emits light at $425 \mathrm{~nm}$ [2]. On the other hand, the indirect CL takes place when the intermediate product electronically excited transfers energy to a suitable acceptor (fluorophore) (Scheme 1b). The relaxation of the fluorophore generates a light emission (Scheme 1c) [3]. This phenomenon

Abel J. Duarte

ajd@isep.ipp.pt

1 Department of Chemical Engineering, REQUIMTE/LAQV, School of Engineering - Polytechnic of Porto, Rua Dr. António Bernardino de Almeida 431, 4200-072 Porto, Portugal

2 Universidade de Trás-os-Montes e Alto Douro, P.O. Box 1013 5000-911 Vila Real, Portugal of emission radiation has recently been proved a powerful tool in areas as distinct as, biotechnology, industry, medicine and water treatment $[1,4]$.

CL methodologies based on oxidative reaction between luminol and hydrogen peroxide have been widely used as sensitive analytical techniques in inorganic chemistry $[5,6]$, in pharmaceutical analysis $[7,8]$, clinical $[9,10]$ and nanotechnology [11] applications. Furthermore, when in presence of an excess of luminol and hydrogen peroxide, the CL intensity is directly proportional to the analyte concentration over several orders of magnitude, which leads to the development of accurate analytical procedures. The attractiveness of the reported CL techniques lies on their simple, fast, high sensitivitive, accuracy, low cost of instrumentation and maintenance, and the fact that it does not require a background light. Although, these methodologies allow detection limits (LOD) at the micromolar range, it is easily possible to decrease at nanomolar levels [12]. An example of such a system that has been used for some authors uses bis-(2,4,6-trichlorophenyl) oxalate (TCPO) [13, 14] for the analytical determination of vitamin $\mathrm{C}$ and vitamin B6. Briefly, the reaction between TCPO and $\mathrm{H}_{2} \mathrm{O}_{2}$ was able to transfer energy to fluoresce indolizine derivatives, via the formation of dioxetanedione intermediate. Nonetheless the use of the TCPO with a fluorophore as, as far as the authors knowledge, never been tested.

In this work, it is reported, for the first time, the CL of TCPO using the luminol as fluorophore using a simple, fast process that does not require expensive equipment. Spectrophotometric techniques were used in order to study this new reaction. 
Scheme 1 Typical CL reaction in the presence of hydrogen peroxide for the oxidation of (a) luminol and (b) TCPO (c) energy transfer to the fluorophore a)<smiles>CC(C)c1ccc(C(=O)[O-])c(N)c1</smiles>

b)<smiles>O=C(Oc1c(Cl)cc(Cl)cc1Cl)C(=O)Oc1c(Cl)cc(Cl)cc1Cl</smiles>

c)

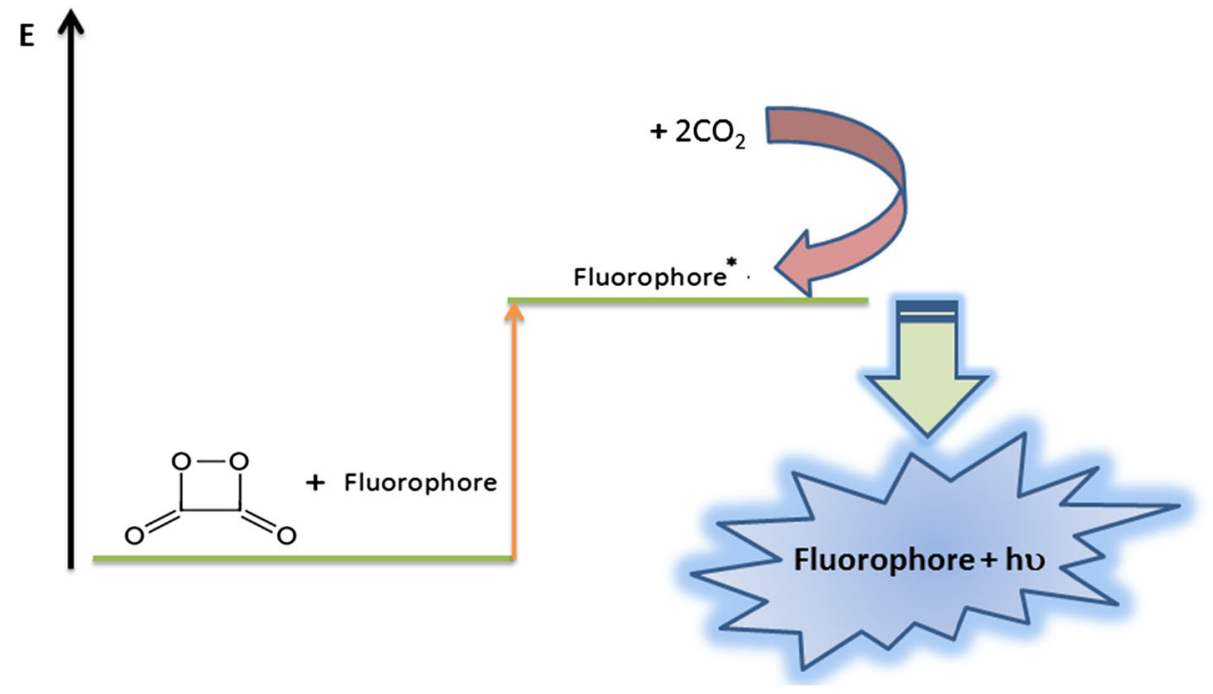

\section{Experimental}

\section{Apparatus}

All chemiluminescence spectra for TCPO reaction were recorded with an homemade setup. The reaction were performed in a $1 \times 10 \mathrm{~cm}$ test tube attached to a support made of matte black PVC sealed to the outside light. The light produced by the CL was conducted by a $1 \mathrm{~mm}$ glass optical fiber to the CCD detector USB2000 from OceanOptic. The spectra were viewed and stored on a PC that communicates with the detector through a USB port. All spectra were recorded with an integration time of $150 \mathrm{msec}$ and 10 scans to average.

Reagents Hydrogen peroxide $(33 \% \mathrm{~m} / \mathrm{V})$, Oxalyl Choride (98\%), Toluene (Sulphure free), Triethylamine pure, Hexane, Rhodamine B, Luminol (98\%), 2,4,6Trichlorophenol, Sodium acetate and Ethyl acetate from Sigma-Aldrich (Madrid, Spain).

\section{Procedures}

TCPO Synthesis According to Mohan's suggestion [15], with some adaptation, $0.395 \mathrm{~g}$ of 2,4,6-trichlorophenol was dissolved in $3 \mathrm{~mL}$ of dry toluene in a round-bottomed flask with a magnetic stirring bar to promote the stirring for $5 \mathrm{~min}$. Then, $0.28 \mathrm{~mL}$ of triethylamine was added, and the flask was put in an ice bath, following the slow addition of $0.10 \mathrm{~mL}$ of oxalyl chloride (during approximately $5 \mathrm{~min}$ ) until the temperature reaches $+8{ }^{\circ} \mathrm{C}$, at this moment a thick-off white precipitate was formed. Then the misture was removed from the ice bath and using an water condenser, the mixture was gently stirred during $30 \mathrm{~min}$ at $65{ }^{\circ} \mathrm{C}$ to complete the reaction. After, the mixture was cooled again in an ice bath, filtered on a Buchner funnel and the formed white solid collected. The solid was washed with a small volume of hexane (to remove the triethylammonium chloride). Then, the dried solid was put in an Erlenmeyer flask and suspended in about $6 \mathrm{~mL}$ of water and using a spatula the lumps of the solid was broken. The 
solid produt was put on the Buchner funnel and washed again with water and dried. At the end, the solid was slowly recrystallized with a minimum volume of boiling toluene and cooled in a ice beath. The solid TPCO was collected using small volumes of hexane.

Chemiluminescence Reaction of the TCPO The fluorophore solution was prepared by dissolving about $6.3 \mu \mathrm{mol}(3 \mathrm{mg}$ of rhodamine B or $1.1 \mathrm{mg}$ of luminol) in $15 \mathrm{~mL}$ of ethyl acetate. In a $1 \times 10 \mathrm{~cm}$ glass tube with magnetic stir bar, it was added $2.7 \mathrm{ml}$ of fluorophore solution, $180 \mathrm{mg}$ of sodium acetate and $144 \mathrm{mg}$ of TCPO. The chemiluminescence was measured when $0.54 \mathrm{~mL}$ of hydrogen peroxide $(33 \% \mathrm{w} / \mathrm{v})$ was added to the mixture.

Control experiments were performed using different chemical qualitative compositions: i) TCPO, luminol and hydrogen peroxide; ii) TCPO, luminol and sodium acetate; iii) TCPO and luminol; iv) TCPO, hydrogen peroxide and sodium acetate; v) TCPO and hydrogen peroxide; vi) Luminol, hydrogen peroxide and sodium acetate; vii) Luminol and hydrogen peroxide.

\section{Results and Discussion}

It is well known that the CL of the TCPO with hydrogen peroxide in the presence of diferents fluorophors compounds, such as 9,10-diphenylanthracene or rhodamine B, originates strong electromagnetic radiation emissions (e.g., maximum wavelength at $600 \mathrm{~nm}$ when the fluorophere is the rhodamine B, Fig. 1 orange). However, in the absence of a fluorophore or an electrolyte (e.g. sodium acetate) the CL reaction does not occur (no light emission), Fig. 1 gray. On the other hand, luminol does not react with

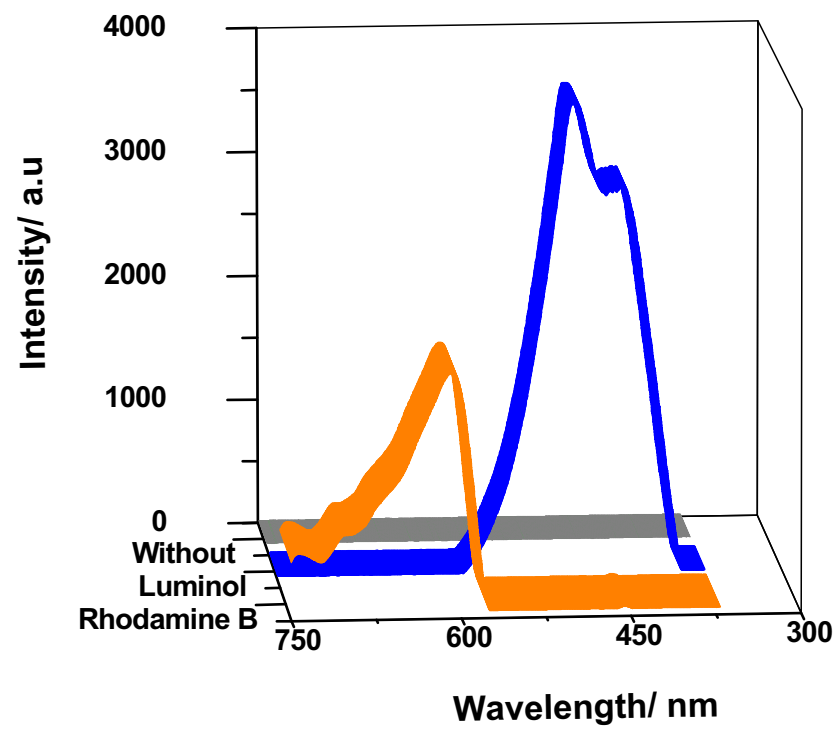

Fig. 1 CL emission intensity of TCPO with hydrogen peroxide without fluophore (gray) and in the presence of luminol (blue) and rhodamine B (orange)

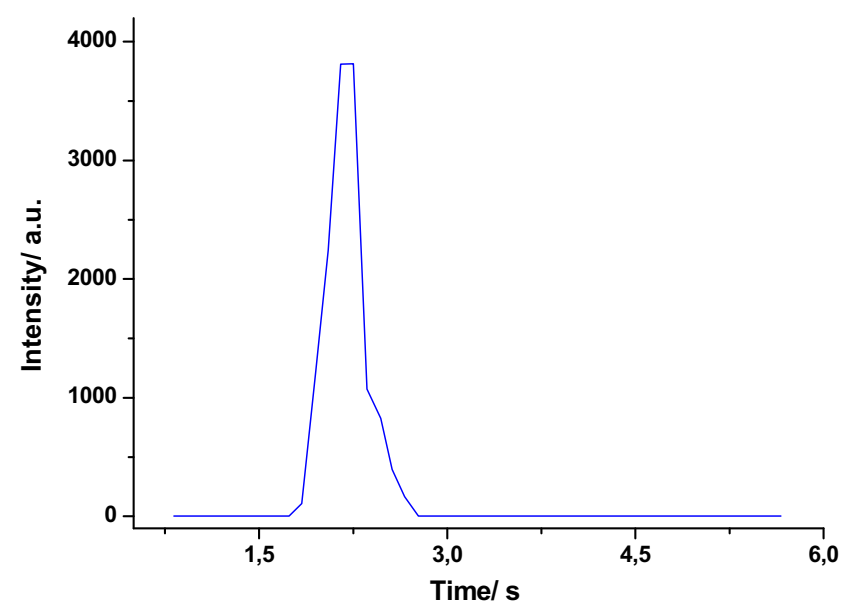

Fig. 2 Effect of the reaction time onto the CL of TCPO in the presence of luminol as fluophore

the hydrogen peroxide (unlike the known TCPO reaction with hydrogen peroxide). This reaction only occurs in the presence of metal catalysts, such as, cobalt (II) or copper (II), organic solvents, such as DMSO, or in the presense of a strong base [16]. However unlike it was typically expected, when we add luminolas a fluorophore in the reactive system composed by TCPO and hydrogen peroxide, an intense and colored electromagnetic radiation is generated, Fig. 1 blue. Moreover, the intensity, duration and color of the TCPO-Luminol system emission is far from negligible, indeed it is quite overwhelming. The obtained CL spectrum of TCPO in the presence of hydrogen peroxide and luminol presented a strong light emission at wavelengths around $440 \mathrm{~nm}$ and another much more intense at $490 \mathrm{~nm}$. This behavior suggests the existence of a more complex mechanism than described in Scheme 1b. This mechanism may produces intermediates derived from luminol and 1,2dioxetanedione which cen be partially responsible for the observed ligh emission. As it is possible to see from the analysis of Fig. 2 the CL of TCPO with luminol-hydrogen peroxide allows a fast reaction system which can be of major importance and vantageous for the development of sensor devices, namely for the detection of contaminants, metals, vitamins and biological molecules (proteins and DNA) in food, environmental and biological samples No light emission was observed in all control experiments.

At this moment, a serie of experiences are underway in order to elucidate the mechanism behind the studied CL reaction. The individual contribution of each chemical and also the respective synergies between then (TCPO, hydrogen peroxide, and luminol), such as, concentraction, molar ratio, and reaction time used in this CL reaction are in study, as well as, the investigation of the chemical intermediates produced during this organic reaction in order to understand all the steps generated in this chemical mechanism. Moreover, it is also intended to use other chemicals in order to discover other fluophores with good CL proprieties. 
However, it is important to take in mind that due to its exuberant CL, this described reaction is an excellent candidate for the development of easy, accurate and sensible miniatured analytical methods [17].

\section{Conclusion}

Yes, luminol has a fluorophore behaviour and in this short communication, it was described for the first time a new CL system based on TCPO- $\mathrm{H}_{2} \mathrm{O}_{2}$ using luminol as fluorophore. In this new system, an intense, strong and light emission at 440 and $490 \mathrm{~nm}$ was generated. Actually, when it was used a molar ratio of TCPO: $\mathrm{H}_{2} \mathrm{O}_{2}$ : luminol of 1: 18: 0.020 more than $90 \%$ of the total emitted light was generated during the first second of the $\mathrm{CL}$ reaction. Moreover, even using small amounts of TCPO (0.32 mmol), the light emission is so strong that the CL was visible to the naked eyes. Considering the fast and strong $\mathrm{CL}$ reaction between TCPO- $\mathrm{H}_{2} \mathrm{O}_{2}$ and luminol, this system can be an excellent candidate to the development of chemical sensors for application in food, environment and health fields for the detection of several compounds, such as, contaminants, metals, antioxidants or biological analytes as DNA.

Acknowledgements This work received financial support from the European Union (FEDER funds through COMPETE) and National Funds (FCT - Fundação para a Ciência e a Tecnologia) through project UID/QUI/50006/2013.

Abbreviations $C L$, Chemiluminescence; TCPO, bis-(2,4,6trichlorophenyl) oxalate

\section{References}

1. Farrell RE (2010) Principles of Detection. In: RNA methodologies (fourth edition). Academic Press, pp 301-320. https://doi.org/10. 1016/B978-0-12-374727-3.00014-0

2. Baj S, Krawczyk T (2006) An investigation into the reaction of hemin-catalysed luminol oxidation by peroxy compounds. J Photochem Photobiol A 183(1-2):111-120. https://doi.org/10. 1016/j.jphotochem.2006.03.002

3. Chaichi MJ, Azizi SN, Heidarpour M, Aalijanpour O, Qandalee M (2012) Chemiluminescence characteristics of furan derivatives as blue Fluorescers in Peroxyoxalate-hydrogen peroxide system. J Fluoresc 22(5):1209-1216. https://doi.org/10.1007/s10895-0121060-2

4. Aslan K, Gueddes CD (2009) Metal-enhanced chemiluminescence. Advanced chemiluminescence concepts for the 21th century. Chem Soc Rev 39:2556-2564. https://doi.org/10.1039/b807498b

5. Kim KM, Kim YH, Oh S, Lee SH (2013) A chelate complexenhanced luminol system for selective determination of co(II),
Fe(II) and $\mathrm{Cr}(\mathrm{III})$. Luminescence 28:372-377. https://doi.org/10. 1002/bio.2392

6. Algi MP, Oztas Z, Tirkes S, Cihaner A, Algi F (2017) Atomistic engineering of chemiluminogens: synthesis, properties and polymerization of 2,3-dihydro-pyrrolo[3,4-d]pyridazine-1,4-dione scaffolds. J Fluoresc 27(2):509-519. https://doi.org/10.1007/s10895016-1978-x

7. Iranifam M (2013) Revisiting flow-chemiluminescence techniques: pharmaceutical analysis. Luminescence 28:798-820. https://doi. org/10.1002/bio.2441

8. Li J, Zhao J, Li S, Zhang L, Lan C, Huang Y, Zhao S (2017) A novel dual target simultaneous chemiluminescence signal amplification strategy for enhancing sensitivity of multiple biomolecule detection. Anal Methods 9:6785-6790. https://doi.org/10.1039/ C7AY2001J

9. Khan P, Idrees D, Moxley MA, Corbett JA, Ahmad F, von Figura G, Sly WS, Waheed A, Hassan MI (2014) Luminolbased chemiluminescent signals: clinical and non-clinical application and future uses. Appl Biochem Biotechnol 173(2): 333-355. https://doi.org/10.1007/s12010-014-0850-1

10. He L, Peng ZW, Jiang ZW, Tang XQ, Huang CZ, Li YF (2017) Novel iron(III)-based metal-organic gels with superior catalytic performance toward Luminol Chemiluminescence. ACS Appl Mater Interfaces 9(37):31834-31840. https://doi.org/10.1021/acsami. $7 \mathrm{~b} 08476$

11. Huang X, Ren J (2012) Nanomaterial-based chemiluminescence resonance energy transfer: a strategy to develop new analytical methods. Trends Anal Chem 40:77-89. https://doi.org/10.1016/j. trac.2012.07.014

12. Marquette CA, Blum LJ (2006) Applications of the luminol chemiluminescent reaction in analytical chemistry. Anal Bioanal Chem 386(3):546-554. https://doi.org/10.1007/ s00216-006-0439-9

13. Tsunoda M, Imai K (2005) Analytical applications of peroxyoxalate chemiluminescence. Anal Chim Acta 541:13-23. https://doi.org/ 10.1016/j.aca.2004.11.070

14. Chaichi MJ, Khajavand T, Mehrzad J, Asghari S, Qandalee M (2013) Indirect Chemiluminescence-based determination of Catecholamines in pharmaceutical formulations by Furandicarboxylate derivative as a novel blue Fluorescer in Peroxyoxalate-H2O2 system. Anal Sci 29:815-821. https://doi. org/10.2116/analsci.29.815

15. Mohan AG, Turro NJ (1974) A facile and effective chemiluminescence demonstration experiment. J Chem Educ 51(8):528. https:// doi.org/10.1021/ed051p528

16. Zhang CX, Li Y, Han S, Dub Y, Liu H (2018) A nitrogen doped carbon quantum dot-enhanced chemiluminescence method for the determination of $\mathrm{Mn}^{2+}$. Anal Methods 10(5):541-547. https://doi. org/10.1039/C7AY02806G

17. Akyazi L, Basabe-Desmonts L, Benito-Lopez F (2018) Review on microfluidic paper-based analytical devices towards commercialisation. Anal Chim Acta 1001:1-17. https://doi.org/10. 1016/j.aca.2017.11.010 